Onkologie 1995;18:384-386

\title{
Contents, Vol. 18, No. 5, 1995
}

Inhalt

Review Articles

Übersichtsarbeiten

Spontaneous Remission in Cancer

Heim, M. E, Köbele, C 388

Methods for Detection of Micrometastatînc Carcinoma Cells in Bone Marrow, Blood and Lymph

Nodes

Pantel, K., Riethmüller, G 394

Monoclonal Antibodies in the Therapy of Minimally Residual Solid Tumors

Schlimok, G., Riethmüller, G. 403

Recent Advances in Anticancer Drug Development

Hanauske, A.-R 410

Dose Intensity of Chemotherapy in Breast Cancer. Results of Clinical Studies

Kroger, N., Zschaber, R., Krüger, W., Zeller, W., Mross, K.,

Zander, A., Hossfeld, D. K 419

Significance of the SCID Mouse Models for the

Study of Normal and Leukemic Human Hemopoiesis and

Gene Therapy

Vormoor, J., Ritter, X, Jürgens, H., Dick, J.E

Spontanremission bei Krebs

Heim, M.E., Köbele, C 388

Methoden zum Nachweis von mikrometastatischen Karzinomzellen in Knochenmark, Blut und Lymphknoten

Pantel, K., Riethmüller, G 394

Monoklonale Antikörper in der Therapie der minimal residualen Erkrankung bei soliden

Tumoren

Schlimok, G, Riethmüller, G 403

Neue Wirkstoffe in der Onkologie

Hanauske, A.-R 410

Dosisintensivierung von Chemotherapie beim Mammakarzinom. Ergebnisse von klinischen

Studien

Kroger, N., Zschaber, R., Krüger, W., Zeller, W., Mross, K.,

Zander, A., Hossfeld,D.K 419

Die Bedeutung der SCID-Mausmodelle für die Untersuchung der normalen und leukämischen menschlichen Hämatopoese und die Gentherapie

Vormoor, X, Ritter, X, Jürgens, H, Dick, XE434 
Original Articles

Prospective Randomized Trial of Dichloromethylene Bisphosphonate (Clodronate) in Patients with Multiple Myeloma Requiring Treatment. A Multicenter Study

Heim, M. E., Clemens, M. R., Queißer, W., Pecherstorfer, M.,

Boewer, Ch., Herold, M., Franke, A., Herrmann, Z., Loose, R.,

Edler, L 439

Phase II Study of Subcutaneous Erythropoietin for Treatment of Myelodysplastic Syndromes

(FAB Type I and II): A Trial of the Phase I/II Study Group of the Association for Medical

Oncology of the German Cancer Society

Hartung, G, Weigold, A., Öhl, S., Essers, U, Heim, M.E.,

Fritze, D., Baldus, M., Hoelzer, D., Bremer, K., Quarder, O.,

Edler, L., Queißer, W.451

Interferon $\alpha 2 b$ and Fotemustine in Patients with Disseminated Melanoma. A Multicenter Phase II

Trial of the AIO Phase I/II Study Group of the German Cancer Society

Kleeberg, U. R., Gawlik, Ch., Bröcker, E. B., Boewer, Ch.,

Chatelain, R.P., Queißer, W., Westerhausen, M., Rothe, B.,

Friedrich, G, Edler, L 456

Tolerance of the Novel Chemosensitizer Dexverapamil in Combination with Anthracycline

Chemotherapy: a Prospective Toxicity Analysis in Advanced Gastrointestinal Cancer

Raderer, M., Maca, T., Kastner, X, Kornek, G, Weinlaender, G,

Hejna, M., Tetzner, C, Scheithauer, W. 462

Active Living with Cancer - Results of a Group Support Program for Cancer Patients and Their

Families

Jany, L

Originalarbeiten

Prospektiv randomisierte Studie mit Dichlormethylen-Bisphosphonat (Clodronat) bei Patienten mit behandlungsbedürftigem inultiplem Myelom. Eine Multicenter-Studie

Heim, M.E., Clemens, M.R., Queißer, W., Pecherstorfer, M.,

Boewer, Ch., Herold, M, Franke, A., Herrmann, Z., Loose, R.,

Edler, L 439

Phase-II-Studie mit subkutanem Erythropoetin bei myelodys-plastischen Syndromen (FAB Typ I und II): Eine Untersuchung der Phase-WI-Studiengruppe der Arbeitsgemeinschaft Internistische Onkologie der Deutschen Krebsgesellschaft

Hartung, G, Weigold, A., Öhl, S., Essers, U., Heim, M.E.,

Fritze, D., Baldus, M., Hoelzer, D., Bremer, K., Quarder, O.,

Edler, L., Queißer, W.451

Interferon $\alpha 2 b$ und Fotemustine bei Patienten mit metastasierendem Melanom. Eine MulticenterPhase-II-Untersuchung der AIO-Phase-II-Studiengruppe der Deutschen Krebsgesellschaft

Kleeberg, U. R., Gawlik, Ch., Bröcker, E. B., Boewer, Ch.,

Chatelain, R. P., Queißer, W., Westerhausen, M., Rothe, B.,

Friedrich, G, Edler, L 456

Verträglichkeit des Resistenzmodulators Dexverapamil in Kombination mit Anthrazyklinen: eine prospektive Toxizitätsanalyse bei Patienten mit fortgeschrittenen Gastrointestinalkarzinomen

Raderer, M., Maca, T., Kastner, X, Kornek, G, Weinlaender, G,

Hejna, M., Tetzner, C, Scheithauer, W 462 
Aktiv leben trotz Krebs: Ergebnisse eines Gruppenunterstützungs-programms für Krebspatienten und ihre Familien

Jany, L468

Continued on page 386

Fortsetzung aufSeite 386

Bibliographischer Hinweis: Inhaltsverzeichnisse dieser Zeitschrift erscheinen regelmäßig in current contents ${ }^{\circledR}$ sowie in anderen bibliographischen Diensten.

Oncological Education

Onkologische Fortbildung

New Possibilities in Diagnostics and Therapy of Adrenal Tumors

Mann, K., Eigler, F. W./Leder, L.-D./Hörmann, R., Jockenhövel, E/

Neumann, K., Langer, R./Giebler, R., Scherer, R 474

Neue Möglichkeiten in der Diagnostik und Therapie von Nebennierentumoren

Mann, K., Eigler, E W./Leder, L.-D./Hörmann, R., Jockenhövel, E/

Neumann, K., Langer, R./Giebler, R., Scherer, R 474

Clinical Information

Consensus Statement of CAO, AIO und ARO about Multimodal Therapy in Gastric Cancer

Junginger, Th 488

Klinische Information

Konsensus der CAO, AIO und ARO: Multimodale Therapie bei Magenkarzinom

Junginger, Th 488

Congress Reports

5th Conference on Adjuvant Therapy of Primary Breast Cancer 1995 - Preliminary Highlights and Conclusions of the Consensus Conference

Thürlimann, B 491

Workshop 'Molecular Development of Cancer', Helgoland, June 1995

Herzog, R 494

Kongreßberichte

5. Konferenz zur adjuvanten Therapie bei primärem Brustkrebs 1995 - Vorläufige Höhepunkte und Schlußfolgerungen der Konsensus-Konferenz

Thürlimann, B 491

Workshop «Molekulare Krebsentstehung», Helgoland, im Juni 1995

Herzog, R 494

Book Reviews $\quad 498$

Industrial Forum $\quad 500$

Buchbesprechungen 498

Industrieforum .500

Reports of Oncological Societies 501

Imprint $\quad 383$

Instructions to Authors $\quad 504$ 
Mitteilungen onkologischer Gesellschaften 501

Impressum 383

Hinweise für Autoren 3. Umschlagseite

Contents of Next Issues

Review Articles

Commentary

Malignant Melanoma: Current Therapeutic Concepts

Tilgen, $\mathrm{W}$.

Parameters of Tumor Progression and Metastases in Bladder Carcinomas

Otto, T., Goepel, M, Rübben, H.

Systemic Chemotherapy for Advanced Bladder Carcinoma

Bex, A, Otto, T., Goepel, M., Rübben, H.

Neuroblastoma: Impact of Biological Characteristics on Treatment Strategies

Ambros, P. E, Ambros, I. M., Ladenstein, R., Gadner, H.

Gene Therapy: Approaches for the Treatment of Malignant Gliomas

Schackert, G, Schackert, H. K.

Malignant Gliomas of the Brain and Surgical Limitations

Steinmetz, A., Schackert, G.

Hematopoietic Progenitor Cell Transplantation in Multiple Myeloma

Goldschmidt, H., Hegenbart, U., Haas, R., Hunstein, W.

Essential Thrombocythemia: Current Opinion in Diagnosis, Clinical Course and Treatment

Lengfelder, E., Hochhaus, A., Hehlmann, R.

Prognostic Significance of 'Micrometastases'

Jauch, K.-W., Friess, S., Grützner, U., Heiss, M. M., Funke I.

Polycythemia Vera: Perspectives for Future Clinical Trials

Heimpel, $\mathrm{H}$.

Are There Any Possibilities to Establish Further Trials for Adjuvant Therapy of Renal Cell

Carcinoma?

Bergmann, L. 